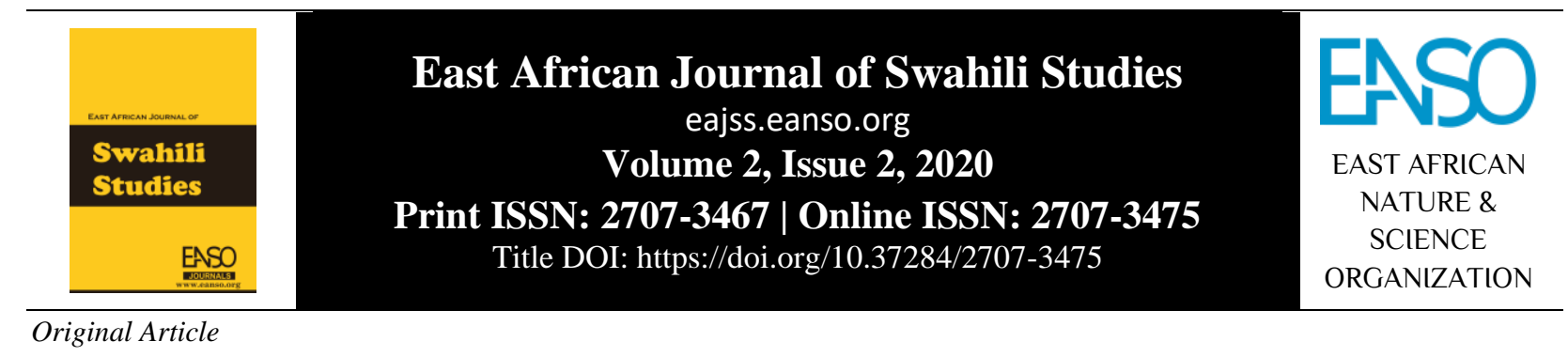

\title{
Mnyambuliko wa Vitenzi Katika Lugha za Kiafrika: Mfano katika Lugha ya Kimasaaba Nchini Uganda
}

\author{
Dr. Willy Wanyenya ${ }^{1 *}$ \\ ${ }^{1}$ Makerere University Business School, P. O. Box 1337, Kampala, Uganda. \\ *Barua pepe ya mawasiliano: willywanyenya@gmail.com \\ ORCID: https://orcid.org/0000-0002-7594-8256
}

DOI ya Nakala: https://doi.org/10.37284/eajss.2.2.184

Tarehe ya Uchapishaji: IKISIRI

15 Juli 2020 Katika makala hii, mtafiti anaeleza jinsi vitenzi vya lugha za Kiafrika vinavyonyambuliwa na kuleta maana tofauti tofauti. Anafanya hivyo kwa

Maneno Muhimu: kurejelea Kimasaaba ambayo ni lugha inayozungumzwa na Wamasaaba

nchini Uganda. Unyambuaji wa vitenzi ni jambo la kawaida na huwa linatekelezwa katika lugha mbalimbali. Hivyo basi, katika makala hii, mtafiti Kiafrika, anaeleza mnyambuliko ya vitenzi katika lugha ya Kimasaaba. Anawasilisha Kimasaaba. mkusanyiko wa vitenzi na kueleza jinsi vinavyonyambuliwa na wazungumzaji. Aidha, mtafiti anaeleza namna vitenzi hivyo vinatumiwa katika sentensi. Mtafiti anafanya hivi kwa sababu lugha nyingi za Kiafrika hazijatafitiwa na kuna mambo mengi sana mazuri ambayo hayajulikani kwa ulimwengu. Katika kazi hii, mtafiti anaandika vitenzi mbalimbali katika lugha asili halafu anavitafsiri katika Kiswahili. Kwenye kazi hii, madhumuni ya utafiti ni kudhihirisha utaratibu wa kunyambua vitenzi katika Kimasaaba. Pili, kueleza maana tofauti zinazotokana na mnyambuliko ya vitenzi. Tatu, kuonyesha kuwa Kimasaaba ni lugha inayoendelea kukua kama lugha zingine za Kiafrika. Mkabala wa utafiti ulikuwa wa nyanjani. Kwa kutumia mkabala huu, mtafiti alizuru nyanjani ambako aliendeleza mahojiano na wahojiwa wake. Majibu ya wahojiwa yalijumlisha data ambazo zilihitajika na mtafiti. Mwishowe, matokeo ya utafiti huu yanaonyesha kuwa Kimasaaba kama lugha nyingine za Kiafrika ina vitenzi vingi sana vinavyonyambuliwa na kwendeleza mazungumzo ya wazungumzaji. 
East African Journal of Swahili Studies, Volume 2, Issue 2, 2020

Article DOI: https://doi.org/10.37284/eajss.2.2.184

\section{APA CITATION}

Wanyenya, W. (2020). Mnyambuliko wa Vitenzi Katika Lugha za Kiafrika: Mfano katkika Lugha ya Kimasaaba Nchini Uganda. East African Journal of Swahili Studies, 2(2), 44-53. https://doi.org/10.37284/eajss.2.2.184

\section{CHICAGO CITATION}

Wanyenya, Willy. 2020. "Mnyambuliko wa Vitenzi Katika Lugha za Kiafrika: Mfano katika Lugha ya Kimasaaba Nchini Uganda”. East African Journal of Swahili Studies 2 (2), 44-53. https://doi.org/10.37284/eajss.2.2.184.

\section{HARVARD CITATION}

Wanyenya, W. (2020) "Mnyambuliko wa Vitenzi Katika Lugha za Kiafrika: Mfano katkika Lugha ya Kimasaaba Nchini Uganda”, East African Journal of Swahili Studies, 2(2), pp. 44-53. doi: 10.37284/eajss.2.2.184.

\section{IEEE CITATION}

W. Wanyenya, "Mnyambuliko wa Vitenzi Katika Lugha za Kiafrika: Mfano katkika Lugha ya Kimasaaba Nchini Uganda", EAJSS, vol. 2, no. 2, pp. 44-53, Jul. 2020.

\section{MLA CITATION}

Wanyenya, Willy. "Mnyambuliko wa Vitenzi Katika Lugha za Kiafrika: Mfano katkika Lugha ya Kimasaaba Nchini Uganda". East African Journal of Swahili Studies, Vol. 2, no. 2, July 2020, pp. 23-31, doi:10.37284/eajss.2.2.184.

\section{UTANGULIZI}

Kitenzi ni neno linaloeleza hali ya kitendo. Kitenzi kimoja kinaweza kunyambuliwa na kudhihirisha hali tofauti za vitendo. Kwa kawaida, kuna hali kama vile hali ya kutenda, kutendeana, kutendwa, kutendeka na kutendesha katika lugha nyingi za Kibantu. Kwa hiyo, katika kazi hii, mtafiti anaorodhesha vitenzi mbalimbali vya Kimasaaba ambayo ni lugha ya Wamasaaba na kuvitafsiri katika Kiswahili. Yafaa ikumbukwe mapema kuwa kuna lahaja tofauti tofauti za Kimasaaba. Kwa hiyo, katika utafiti huu, mtafiti alizingatia lahaja mojawapo ijulikanayo kama 'Lubuuya.' Akisha fasiri vitenzi, mtafiti anaeleza utaratibu wa kuvinyambua. Baada ya kueleza jinsi vitenzi hivyo hunyambuliwa, mtafiti alifafanua namna ambavyo hutumiwa katika sentensi. Mtafiti anafanya hivyo kwa kuzingatia maelezo ya waandishi mbalimbali kuhusu mnyambuliko wa vitenzi. Mwishowe, mtafiti anahitimisha kazi yake kwa kutoa maoni yake kuhusu mambo muhimu yenye uhusiano na utafiti.

\section{SUALA LA UTAFITI}

Kwa mujibu wa mtafiti, kuna makabila mengi sana barani Afrika. Kila kabila lina lugha yake asili. Kwa hiyo, kuna lugha nyingi sana zinazozungumzwa baranani humu. Hata hivyo, sehemu kubwa ya lugha hizo hazijatafitiwa na kuna mambo mengi sana yanayowasilishwa na lugha hizo ambayo bado hayajajulikana kwa ulimwengu. Ni lugha chache sana za Kiafrika ambazo zimeshughulikiwa kitaalamu. Kutokana na upungufu huu, mtafiti aliamua kuendeleza utafiti huu akidhamiria kueleza jinsi mambo yalivyo na Kimasaaba. Hii ni kwa sababu lugha nyingi ndogo ndogo zimeangamia kwa kukosa kufanyiwa utafiti na zingine bado zinaendelea kuangamia.

\section{MBINU ZA UTAFITI}

Katika kazi hii, mtafiti alitumia mbinu ya mahojiano. Kwa kutumia mbinu hii, mtafiti alizuru nyanjani ambako alikutana na wahojiwa wake. Kabla ya mahojiano, mtafiti alieleza wahojiwa wake sababu ya utafiti wake. Baada ya kufanya hivyo, alianza kuendeleza mahojiano na wahojiwa hao. Wahojiwa walijumlisha wanawake 50 na wanaume 50. Mtafiti alihakikisha kuwa kuna idadi sawa ya wanawake na wanaume kutokana na suala la usawa wa kijinsia. Wahojiwa hawa waliteuliwa kwa kutumia mbinu ya sharabera. Kwa kutumia mbinu hii, mtafiti aliendeleza mazungumzo na wahojiwa wake kama alivyokutana nao. Hakutumia utaratibu maalumu kuwateua wahojiwa hao. Mtafiti alikuwa na mwongozo wa maswali ambayo aliyatumia katika mahojiano. Mtafiti alitumia mwongozo ili asije akasahau jambo lolote muhimu. Mijadala iliyoendelezwa ilihusika kwa kiasi kikubwa na mnyambuliko wa vitenzi. Baada ya kutaja kitenzi, mtafiti aliwauliza namna ya kukinyambua katika hali tofauti tofauti. 


\section{MAPITIO YA MAANDISHI}

Kwa mujibu wa Baraka (2018), mnyambuliko wa vitenzi ni jambo muhimu sana katika lugha za Kibantu. Kulingana na maelezo yake, mnyambuliko wa vitenzi hupelekea kitenzi kimoja kuleta maana tofauti tofauti kutokana na hali mbalimbali za mnyambuliko. Baraka (2018) aliendeleza utafiti wake kwa kurejelea lugha ya Kinyakyusa ambayo ni lugha yake ya mama. Anasisitiza kuwa lugha hii ina vitenzi vingi sana vinavyokubali kunyambuliwa. Anaendelea kusema kuwa katika utafiti wake, wazee waliweza kunyambua baadhi ya vitenzi hivyo katika hali mbalimbali kama ilivyo katika majedwali yafuatayo:

Jedwali 1: Minyambuliko ya Vitenzi Katika Hali ya Kutendewa

\begin{tabular}{llll}
\hline Vitenzi Kiswahili & Kinyakyusa & Kauli ya kutendewa & Kiswahili \\
\hline Lala & Lambalal-a & Lambal-elw-a & Lal-i-w-a \\
Fanya & Bhomba & Bomb-elw-a & Fany-i-w-a \\
Suka & Suk-a & Suk-elw-a & Suk-i-w-a \\
Sukuma & Gut-a & Gut-elw-a & Sukum-i-w-a \\
Kata & But-a & But-elw-a & Kat-i-w-a \\
Lima & Lem-a & Lem-elw-a & Lim-i-w-a \\
Ona & Ket-a & Ket-elw-a & On-e-wa \\
Songa & Eng-a & Eng-elw-a & Song-e-w-a \\
\hline
\end{tabular}

Kwa mujibu wa Jedwali 1, data zinaonyesha kuwa Kiswahili kina mofu mbili za kuonyesha kauli ya kutendewa ambazo ni: $\{-\mathrm{i}-\}$ na $\{-\mathrm{e}-\}$ lakini katika lugha ya Kinyakyusa vitenzi vikinyambuliwa vina mofu moja tu ambayo ni $\{$-elw- $\}$. Hali kadhalika, jedwali lifuatalo linaonyesha jinsi vitenzi vya Kinyakyusa vinavyonyambuliwa katika hali ya kutendeana:

\section{Jedwali 2: Mnyambuliko wa vitenzi Katika Hali ya Kutendeana}

\begin{tabular}{lll}
\hline Vitenzi & Vitenzi vya Kinyakyusa & Kaulya Kutendeana \\
\hline Sukuma & Gut-a & Gut-an-a \\
Suka & Suk-a & Suk-an-a \\
Seka & Sek-a & Sek-an-a \\
Vuta & Lus-a & Lus-an-a \\
Kata & But-a & But-an-a \\
Beba & Pemb-a & Pemb-an-a \\
Ruka & Luk-a & Luk-el-an-a \\
Pika & Pej-a & Pej-el-an-a \\
Imba & Emb-a & Emb-an-a \\
Cheza & Kin-a & Kin-el-an-a \\
\hline
\end{tabular}

Kulingana na Jedwali 2, inabainika wazi kuwa kitenzi cha Kinyakyusa kikinyambuliwa huweza kuonyesha hali ya kutendeana. Hii ni hali ya kila mtu kumtendea mwenzake jambo hilo hilo. Yaani, wote wanafanyiana jambo hilo na hii huonyeshwa na kiambishi $\{$-an- $\}$, $\{$-el- $\}$.

Khamis ( 2008) anaeleza kuwa lugha ya Kiswahili, kama ilivyo na lugha nyingi za Kibantu ina utajiri mkubwa sana wa mfumo wa unyambulishaji wa vitenzi. Idadi kubwa ya shughuli za kisarufi ambazo kwa kawaida huwasilishwa kwa njia ya miundo ya kisintakisia katika lugha zenye uhaba wa msamiati, maneno hudhibitiwa kwa utaratibu wa unyambulishaji wa vitenzi. Katika Kiswahili kwa mfano, unyambulishaji hupelekea kiambishi tamati kiwekwe kwenye umbo la kitenzi; kama ifuatavyo:

Chez-a $\rightarrow$ chez- esh $-a$,

Gong-an-a $\rightarrow$ gong-an-ish- $a$

Minyambuliko $\{-$ esh $\}$ na $\{-i s h\}$ imeambishwa kwenye vitenzi ili kuwasilisha dhana ya kitendo. Khamis (2009) anasema kuwa kitenzi ni aina ya 
maneno yanayoelezea tendo linalofanywa na nomino. Anasema mfano wa vitenzi ni kama vile og-a, li-a, sinz-a, n.k. Mifano hii ya vitenzi ina maumbo yenye kuhusisha mzizi mwanzoni na kiambishi cha dhamira dhahiri -a mwishoni. Maumbo kama haya yenye kuhusisha mzizi na kiambishi tamati -a ndiyo maumbo ya asili na ya kimsingi. Maneno haya ndiyo yanajulikana kama vitenzi na hupatikana katika kamusi. Khamis (2009) anaeleza kuwa vitenzi huwa ni maneno yanayowakilisha jambo linalofanywa na nomino. Mifano ya maneno hayo ni: lia, lala, pumua, tembea, kimbia, pika n.k.

Kwa mujibu wa Kihore, Massamba na Msanjila (2003), kitenzi ni neno linaloeleza jambo linalotendwa au lililotendeka. Kitenzi hueleza tendo lililofanyika au litakalofanywa na mnyama, mtu au kiumbe chochote kinachoweza kutenda jambo. Mifano ni: soma, simama, lima, cheza, omba, kimbia, ruka, andika, sikiliza, uliza, shukuru, amuru n.k.

Kihore na wenzake (2003) wanasema kuwa unyambulishaji ni upachikaji wa viambishi kwenye mzizi ili kuunda maneno mapya. Katika lugha ya Kiswahili kuna viambishi nyambulishi ambavyo vinatumika katika uundaji wa vitenzi vipya kama ilivyo hapo chini:
a) pend $+\mathrm{a}$
b) pend $+\mathrm{e}+\mathrm{a}$
c) pend +ele $+\mathrm{a}$
d) pend + ele $+\mathrm{an}+\mathrm{a}$
e) pend $+\mathrm{ez}+\mathrm{a}$
f) pend $+\mathrm{ez}+\mathrm{esh}+\mathrm{a}$
g) pend $+\mathrm{ez}+\mathrm{esh}+\mathrm{an}+\mathrm{a}$

Kutokana na mzizi wa kitenzi pend,-tunaweza kupata maneno kama yale yaliyoorodheshwa hapo juu ambapo viambishi tamati -e, -an, -ele, -esh ni nyambulishi kutokana na kufanya maumbo yanayohusika kuwa vitenzi vyenye maana tofauti. Kiswahili kina viambishi tamati nyambulishi vinane ambavyo huambikwa kwenye mizizi ya vitenzi kwa namna ambayo imeonyeshwa hapo juu. Vinyambulishi hivyo ni kama vifuatavyo: (a). -w (b) -ik / -ek (c) -ish-/-iz-/-esh-/-ez- (d) -il- (e) o-/-u (f)-am- (g) -an- h) -at-

Wahiga (1999) anaeleza kuwa neno 'mnyambuliko' hutokana na vitenzi viwili ambavyo ni 'nyambua' na 'nyumbua.' Kulingana na mtaalamu huyu, kunyambua ni kukata kitu vipande vipande halafu kunyumbua ni kuvuta au kutanua kitu chororo ili kukirefusha. Kwa mfano, tukitaka kuunda kitenzi cha kauli ya kutendesha kutokana na vitenzi vya kauli ya kutenda -piga, lima, cheza, tua- tutaongeza viambishi $\{$ ish $\},\{$ esh $\}$, \{liz\} kati ya kiishio na mzizi wa kila kitenzi. Tazama mifano:

\section{Jinsi ya kutenda}
a) Pig-a
b) Lim-a
c) Chez-a

\section{Jinsi ya kutendesha}

pig-ish-a

lim- ish-a

chez-esh-a
Waihiga (1999) anaeleza kuwa vitenzi ni maneno ambayo kwa kawaida husimama kwa ajili ya kuarifu jambo linalofanywa na nomino au kiwakilisha. Kuhusu mnyambuliko wa vitenzi vya asili ya Kibantu, mwandishi huyu anaeleza neno mnyambuliko kwa kurejelea maneno mawili ambayo ni 'nyumbua' na 'nyambua.' Mtaalamu huyu anasema kuwa kunyambua ni kukata kitu vipande vipande na kunyumbua ni kuvuta au kutanua kitu chororo ili kukirefusha. Anasema kuwa kwa kuvinyambua vitenzi tunapata kauli mbalimbali ambazo ni: kauli ya kutendea, kauli ya kutenda, kauli ya kutendwa, kauli ya kutendesha, kauli ya kutendeka, kauli ya kutendana na kauli ya kutendeana.

Katamba (2003) anaeleza kuwa unyambulishaji, kama unavyojidhihirisha katika lugha za Kibantu na lugha nyinginezo, ni mchakato unaozalisha aina mpya za maneno au unaoongeza maana fulani kwenye mzizi au shina. Kwa mfano, kutokana na mzizi pig-, tunapata neno 'piga' ambalo ni kitenzi. $\mathrm{Na}$ tukilinyambua neno hili tunapata maneno kama vile pigana, piganisha, pigika ambayo yana maana tofauti.

TUKI (2004) wanaeleza kuwa uambishaji ni utaratibu wa kuweka viambishi kwenye vitenzi na kuleta maana tofauti tofauti. Tendo hili ndilo linaitwa unyambulishaji wa vitenzi. Kwa mujibu wa wataalamu hawa, kitenzi kina sehemu kuu ambayo ni mzizi unaokubali kuongezwa kwa viambishi mwanzoni au mbeleni. Matei (2008) anasema kuwa katika aina zote za maneno, kitenzi ndicho kinachoonyesha tendo lililofanywa au linalofanywa. Anaendelea kueleza kuwa kitenzi 
kinaweza kudhihirisha hali mbalimbali kutokana na unyambulishaji. Ameweza kugawa vitenzi katika makundi mawili yaani vitenzi vya kutenda na vitenzi vya kuwa na kitu.

Kihore na wenzake (2003) wanagawa vitenzi katika aina kuu tatu ambazo ni; kitenzi kikuu(T), kitenzi kisaidizi (Ts) na kitenzi kishirikishi (t). Wanaeleza kuwa kitenzi kikuu ni kitenzi ambacho kinaweza kujitokeza peke yake katika sentensi na kikakamilisha maana. Mfano; analala, tutampiga Juma, Khamis atamwona Musa kesho. Kitenzi kisaidizi ni kitenzi ambacho kinaandamana na kitenzi kikuu katika sentensi kama ilivyo katika sentensi zifuatazo: alikuwa analala, tunapenda kula, Juma alitaka kutudhulumu mali yetu. Mifano hii inaonyesha kuwa vitenzi visaidizi huwa vinatangulia kisha vitenzi vikuu vikafuata.

Kitenzi kishirikishi, kiambishi kile kile ambacho umbo lake ni lile la "ni" au "si" kinachukuliwa kuwa kinashirikisha vipashio vingine katika sentensi. Mfano; Musa ni mwalimu, mji wa msoma ni mdogo kuliko mji wa mwanza, huyu kijana si mwadilifu, mimi si mwanafunzi. Kutokana na muktadha wa sentensi hizi, inaelekea kuwa vipashio hivi huchukuliwa kuwa vinashirikisha vipashio vya upande wa kushoto na vile vya kulia wa kitenzi chenyewe. Sentensi hizo zipo kwenye njeo ya wakati uliopo. Sentensi hizi zikibadilishwa kuingia katika njeo tofauti, vitenzi vishirikishi vilivyomo ndani yake (yaani "ni" na "si") hubadilika na kupata umbo la kitenzi "kuwa," tazama mifano hii: Musa alikuwa mwalimu, mji wa msoma ulikuwa mdogo kuliko mji wa mwanza, huyu kijana hakuwa mwadilifu, mimi sikuwa mwanafunzi.

Kulingana na wataalamu hawa, kitenzi ni neno linaloeleza kitendo. Kitenzi ndicho hubeba nomino na kuonyesha inataka kufanya nini na kwa wakati gani. Lakini unyambulishaji ni ile hali ya kuliongezea neno uwezo wa kutumika katika hali mbalimbali na kuweza kuzalisha msamiati mpya tofauti na ule wa awali japo mzizi wake unabaki hivyo hivyo. Pia, unyambulishaji ni maneno yenye mzizi sawa lakini huweza kujipambanua katika maumbo tofauti na kuweza kuzalisha msamiati mpya.

Kulingana na Mhadhara wa 8 katika kitabu kilichotolewa na Chuo Kikuu Huria cha Tanzania mwaka 2008, mnyambuliko ni neno ambalo maana yake inatokana na maana ya maneno mawili: (i) Kunyambua, linalomaanisha kata vipande vipande; (ii) Kunyumbua, likimaanisha vuta na kutanua kitu chororo, mfano kama kamba ya mpira. Katika sarufi, mnyambuliko ni ule uwezo wa mzizi au shina kuambishwa mofu mwanzoni au mwishoni mwake. Matokeo yake ni kupanua maana ya neno husika. Hii ni kazi ya kila kiambishi - kujenga mizizi mipya na mashina mapya ya maneno ambayo huitwa mashina au mizizi ya mnyambuliko. Tunaweza kusema pia kuwa hii ni kazi ya viambishi vya mnyambuliko au viambishi vijenzi. Aghalabu, viambishi vijenzi hutokea mwishoni mwa shina au mzizi. Na kwa mantiki hiyo, vimeendelea kuitwa viambishi tamati-vijenzi. Kwa mantiki hiyo, mnyambuliko unahusisha viambishi vya aina mbili: (a) Viambishi-Maana, ambavyo hutokea mwanzoni mwa mzizi wa neno. Viambishi hivi vinaweza kutanua maana ya neno bila kubadilisha mzizi wa neno. Kwa mfano: tu-tam-pig-an-ish-a.

Tunaona hapa kwamba: (i) -pig- ni mzizi wa neno tutampiganisha. (ii) tu-ta-m- ni viambishi vya mwanzoni mwa mzizi -pig- (iii) -an-ish-a ni viambishi vya mwishoni mwa mzizi -pig-. Viambishi vya mwanzoni na vile vya mwishoni mwa mzizi -pig- vinaleta maana tofauti tofauti kama ilivyo hapa: (i) Kiambishi tu- ni cha nafsi ya kwanza wingi (sisi). (ii) Kiambishi -ta- ni cha njeo ijayo. (iii) Kiambishi -m- ni cha kumrejesha mtendwa, nafsi ya tatu, umoja (yeye). (iv) Kiambishi -an- ni cha kutendeana. (v) Kiambishi ish-ni cha kutendesha. (vi) Kiambishi -a- ni cha kutamatisha kauli (b) Viambishivijenzi/mnyambuliko hutokea mwishoni mwa shina la neno.

Viambishi hivi vina kazi tatu: (i) kutanua maana ya neno (ii) kujenga mzizi au shina la mnyambuliko (iii) Kubadilisha neno kutoka aina moja na kuingia katika aina nyingine ya neno. Kwa mfano: (i) kuomba = omboleza =ombolez $-(=$ mzizi wa mnyambulisho) $=$ ombi, ombolezo (= majina) (ii) ku-sifu = sifia; sifiana. $=$ sifa, wasifu (= majina). Aghalabu, viambishi-vijenzi hutokea mwishoni mwa shina na kwa mantiki hiyo, huitwa viambishi tamati-vijenzi. 
Kwa mujibu wa Niyirora na Ndayambaje (2012), unyambulishaji wa vitenzi ni utaratibu wa uundaji wa vitenzi vipya katika lugha kwa kuongeza viambishi nyambulishi kwenye maumbo ya mizizi. Wataalamu hawa wanasema kuwa katika lugha ya Kiswahili kuna viambishi nyambulishi ambavyo vinatumika katika uundaji wa vitenzi vipya kama inavyoonekana katika mifano ifuatayo: (a) (pend+a) (pend+e+a) (pend+ele+a) (pend+ele+an+a) (pend+ez+a) (pend+ez+esh+ag) (pend+ez+esh+an+a).

Katika mifano hii, kutokana na mzizi wa kitenzi pend-, tunaweza kupata maneno kama yale yaliyoorodheshwa katika mifano ambapo viambishi tamati -e, -an, -ele-, -esh, ni nyambulishi kutokana na kufanya maumbo yanayohusika kuwa vitenzi vyenye maana tofauti. Katika Kiswahili kuna viambishi nyambulishi vinane ambavyo huambikwa kwenye mizizi ya vitenzi ambavyo ni pamoja na: -w-, -ik-/-ek-, - ish/-iz-/-esh-/-ez-, -il/-el- n.k. Viambishi hivyo huonyesha hali ya vitenzi:

1. Hali ya kutendua: Hii hali inayonyesha kinyume cha kitenzi kinachotajwa Mfano: tendua, anua, fungua, fukua, tatua

2. Hali ya kutendewa: Mtu aliyefinyiwa jambo fulani ndiye anayezungumza mfano : chumbiwa, pigiwa, adhibiwa, imbiwa.

3. Hali ya kutendwa: Hapa mtu mtendwa ndiye huwa amefanyiwa jambo fulani. Mfano: pigwa, liwa, chorwa, vunjwa, katwa.

4. Hali ya kutendeka: Hapa vitenzi hueleza kuwepo kwa uwezo wa kitendo cha kitenzi kutokea, mfano: someka, imbika, ombeka, fungika.

5. Hali ya kutendeana: Katika hali hii ya mnyambuliko, mtu anamtendea mwingine jambo naye huyo aliyefanyiwa akamfanyia mwenzake kitendo hicho hicho, mfano: katiana, vunjiana, simamiana, tendeana.

6. Hali ya kutendesha: Hali hii ya mnyambuliko huleta maana ya kusababisha. Kwa hiyo, vitenzi vyote katika hali hii huonyesha kuwa vimesababishwa na hali, kitu ama mtu fulani. Kwa mfano, katika sentensi zifuatazo:
a) Niliwapiganisha.
b) Tuliwapikisha.
c) Tuliwagombanisha.
d) Aliwaimbisha.
e) Aliwasomesha.

Kwa mujibu wa Kihore na wenzake (2012), unyambulishaji ni upachikaji wa viambishi kwenye mzizi ili kuunda maneno mapya. Wanaendelea kusema kuwa katika lugha ya Kiswahili kuna viambishi nyambulishi ambavyo hutumika katika uundaji wa vitenzi vipya. Kihore na wenzake (2012) wanaendelea wanafafanua unyambulishaji wa vitenzi kama utaratibu wa uundaji wa vitenzi vipya katika lugha kwa kuongeza viambishi nyambulishi kwenye maumbo ya mzizi. Kulingana nao, utaratibu huu hufanyika katika hatua kama vile:
(a) Pig-w-a
(b) Pig-an-a
(c) Pig-i-a
(d) Pig-i-an-a
(e) Pig-i-w-a
(f) Pig-an-ish-a

Mbwillow (2017) anaeleza kuwa maneno ya lugha asilia ya binadamu huundwa kwa njia mbalimbali. Anaeleza njia kama vile, uradidi, kubuni, uambatanishaji na uambatizi kwa maana ya uambishaji na unyambulishaji. Anaeleza kuwa uambikaji ni kitendo cha upachikaji au uongezaji wa mofu au viambishi kabla au baada ya mzizi wa neno, kiini au shina la neno. Anasisitiza kuwa njia kuu ya uundaji wa maneno ni uambishaji na unyambulishaji. Anafafanua unyambulishaji kama mchakato wa upachikaji wa mofu/viambishi baada ya mzizi wa neno na shina. Katika kazi yake, anaeleza kuwa uambishaji na unyambulishaji huunda maneno mapya. Anaeleza kuwa uambishaji na unyambulishaji unaweza kutobadili kategoria ya neno bali zikatanua maana ya neno husika.

Mbwillow (2017) anaendelea kueleza kuwa unyambulishaji huleta kauli mbalimbali. Katika maelezo yake anataja kauli ya kutenda, kutendewa, kutendeka na kutendeana. Anatoa mfano wa neno 'lima' na kufafanua jinsi linavyonyambuliwa; limi-a (kauli ya kutendea), lim-w-a (kauli ya kutendwa), lim-ik-a (kauli ya kutendeka), lim-i-ana (kauli ya kutendeana). Mbwillow (2017) anaeleza kuwa si kila neno au kategoria ya maneno ya Kiswahili hukubali uambishaji au unyambulishaji. Anasema kuwa si rahisi vitenzi vyote vya Kiswahili kuambishwa. Kwa mujibu wa mtaalamu huyu, kuna 
baadhi ya vitenzi katika lugha ya Kiswahili ambavyo haviambishwi.

Kwa mujibu wa Paneli la Kiswahili (2019), mnyambuliko wa vitenzi ni jinsi vitenzi vinavyobadilika kulingana na kauli/hali mbalimbali. Kwa mfano, kutokana na kitenzi 'soma' tunaweza kupata: somea, somewa, somwa n.k. Wanasema ni vizuri kuelewa kwamba sio vitenzi vyote vinanyambulika katika kila kauli. Kulingana na Paneli hili, kuna kauli zifuatazo za minyambuliko: Kauli ya Kutenda - kitendo katika hali yake ya kawaida (bila kunyambuliwa), Kauli

hedwali 3: Namna vitenai vinavyobadilika kulinga ya Kutendea - kufanya kitendo kwa niaba ya mtu mwingine, Kauli ya Kutendana - unamfanya mtu kitendo naye anakufanya vivyo hivyo, Kauli ya Kutendeana - unamfanyia mtu kitu kwa moyo safi naye anakufanyia, Kauli ya Kutendwa - kuathirika moja kwa moja na kitendo, Kauli ya Kutendewa kitendo kufanywa kwa niaba yako au kwa ajili yako, Kauli ya Kutendeka - kitendo kukamilika au kuwa katika hali timilifu, Kauli ya Kutendesha kumfanya mtu atende jambo fulani na Kauli ya Kutendeshana - mtu anakufanya utende jambo fulani, nawe unamfanya atende jambo lilo hilo.

\begin{tabular}{lllllllll}
\hline Tenda & Tendea & Tendana & Tendeana & Tendwa & Tendewa & Tendeka & Tendesha & Tendeshana \\
\hline Fanya & Fanyia & Fanyana & Fanyiana & Fanywa & Fanyiwa & Fanyika & Fanyisha & Fanyishana \\
\hline Lima & Limia & Limana & Limiana & Limwa & Limiwa & Limika & Limisha & Limishana \\
\hline Pika & Pikia & Pikana & Pikiana & Pikwa & Pikiwa & Pikika & Pikisha & Pikishana \\
\hline Lia & Lilia & ? & Liliana & $?$ & Liliwa & Lilika & Liza & Lizana \\
\hline Kula & Lia & Lana & Liana & Liwa & Liwa & Lika & Lisha & Lishana \\
\hline Penda & Pendea & Pendana & Pendeana & Pendwa & Pendewa & Pendeka & Pendeza & Pendezana \\
\hline Omba & Ombea & Ombana & Ombeana & Ombwa & Ombewa & Ombeka & Ombeza & Ombezana \\
\hline
\end{tabular}

\section{MTAZAMO WA MTAFITI KUHUSU MAPITIO YA MAANDISHI}

Maandiko ya wataalamu mbalimbali, yanadhihirisha kuwa kitenzi ni neno linaloeleza kitendo ilhali mnyambuliko ni hali ya kuwekea kitenzi viambishi na kuzalisha maneno mapya yenye maana tofauti tofauti. Maandishi mbalimbali yanaonyesha pia kuwa kuna hali mbalimbali za mnyambuliko wa vitenzi. Hali hizo mbalimbali zinajumlisha hali ya kutenda, hali ya kutendea, hali ya utendeana, hali ya kutendeka, hali ya kutendesha na hali ya kutendeshana. Hali kadhalika, maandishi yaliyosomwa yanaonyesha kuwa si vitenzi vyote katika lugha huwa vinanyambuliwa-kuna vitenzi vinavyonyambuliwa na kuna vile visivyonyambuliwa.

Maandishi yanaonyesha pia kuwa kuna wasomi wengi sana ambao wameandika kuhusu mnyambuliko wa vitenzi katika lugha. Kuna wasomi ambao wameandika kuhusu mnyambuliko wa vitenzi katika lugha ya Kiswahili na kuna wale ambao wameandika kuhusu mnyambuliko wa vitenzi katika lugha zingine Kiafrika kama vile: Kinyakyusa, Kigiryama, Kigichugu nk. Hata hivyo, hakuna ushahidi kuwa kuna mtaalamu yeyote ambaye ameandika kuhusu mnyambuliko wa vitenzi katika Kimasaaba.

\section{MATOKEO YA UTAFITI}

\section{Jedwali 4: Hali Mbalimbali za Minyambuliko ya Vitenzi Katika Kimasaaba}

\begin{tabular}{llllll}
\hline Kutenda & Kutendea & Kutendeana & Kutendwa & Kutendeka & Kutendesha \\
\hline Khupa/ & Khupila/ & Khupana/ & Khupikha/ & Khupikhikha/ & Khupanisa/ \\
Piga & Pigia & Pigana & Pigwa & Pigika & Piganisha \\
\hline Tekha/ & Tekhela/ & Tekhanila/ & Tekhikhila/ & Tekhikha/ & Tekhisa/ \\
Pika & Pikia & Pikania & Pikiwa & Pikika & Pikisha \\
\hline Lomisa/ & Lomisila/ & Lomisana/ & Lomisikha/ & Lomisikhikha/ & Lomisanisa/ \\
Gombana & Gombania & Gombana & Gombanwa & Gombanika & Gombanisha \\
\hline Khwala/ & Khwalila/ & Khwalana/ & Khwalikha/ & Khwalikhikha/ & Khwalikhisisa/ \\
\hline
\end{tabular}


East African Journal of Swahili Studies, Volume 2, Issue 2, 2020

Article DOI: https://doi.org/10.37284/eajss.2.2.184

\begin{tabular}{llllll}
\hline Kutenda & Kutendea & Kutendeana & Kutendwa & Kutendeka & Kutendesha \\
\hline Oa & Olea & Oana & Olewa & Oleka & Oza \\
\hline Sheeba/ & Sheebela/ & Shebanila/ & Shebikhila/ & Shebikhikhila/ & Shebisisa/ \\
Fyeka & Fyekea & Fyekania & Fyekiwa & Fyekikishika & Fyekisha \\
\hline Kangulula/ & Kangululila/ & Kangululana/ & Kangululikha/ & Kangululikhikha/ & Kangululisa/ \\
Fungua(Jela) & Fungulia & Funguana & Funguliwa & Funguka & Fungulisha \\
\hline Buwa/ & Bweila/ & Buwana/ & Bweikha/ & Bweikhikha/ & Bweisisa/ \\
Funga(Jela) & Fungia & Fungana & Fungwa & Fungika & Fungisha \\
\hline Yikala/ & Yikalila/ & Yikalanila/ & Yikalikhila/ & Yikalikhikhila/ & Yikalisisa/ \\
Funga(Mlango) & Fungia & Fungania & Fungiwa & Fungikiwa & Fungisha \\
\hline Yikula/ & Yikulila/ & Yikulanila/ & Yikulikhila/ & Yikulikhikhila/ & Yikulisisa/ \\
Fungua(Mlango) & Fungulia & Funguania & Funguliwa & Fungulikikiwa & Fungulisha \\
\hline Bukula/ & Bukulila/ & Bukulana/ & Bukulikha/ & Bukulikhikha/ & Bukulisisa/ \\
Nyakua & Nyakulia & Nyakuana & Nyakuliwa & Nyakuligika & Nyakulisha \\
\hline Kuta/ & Kutila/ & Kutana/ & Kutiwa/ & Kutikha/ & Kutisisa/ \\
Seng'enya & Seng'enyea & Seng'enyana & Seng'enywa & Seng'enenyika & Seng'esha \\
\hline
\end{tabular}

Tukitazama Jedwali 4, tunaona kuwa Kimasaaba huwa kinanyambuliwa kama lugha zingine za Kiafrika kwa kuongezea vitenzi viambishi. Minyambuliko yenyewe huchukua hali za kutenda, kutendea, kutendeana, kutendwa, kutendeka na kutendesha. Kwa kuzingatia jedwali tunaona kwamba vitenzi katika Kimasaaba vikiongezewa viambishi huwa na maana tofauti. Kutokana na minyambuliko, kitenzi kimoja hutumika katika sentensi tofauti na kuleta maana tofauti. Tazama mifano katika sentensi kwa kutumia kitenzi 'khupa/piga': Mnyambuliko wenyewe umezingatia lahaja mojawapo ya Kimasaaba inayojulikana kama 'Lubuuya.'

\section{Khukhola/Hali ya Kutenda}

Katika hali ya kutenda, kiishio cha kitenzi cha Kimasaaba huwa kinabadilika na kuwa -ile. Tazama mifano:

1. Na+mu+khup+il+e: Namukhupile/Nilimpiga (Na- nafsi ya kwanza umoja, -mu- kiambishi cha mtendewa, -khup- ni mzizi wa kitenzi, -ilni kinyambulishi, -e ni kiambishi tamati).

2. Khwa+ba+khup+il+e: khwabakhupile/Tuliwapiga (Khwa- nafsi ya kwanza wingi, -ba kiambishi cha watendewa, khup- ni mzizi wa kitenzi, -il- ni kinyambulishi, -e ni kiambishi tamati).

3. Wa+mu+khup+il+e: wamukhupile/ulimpiga (Wa- nafsi ya pili umoja, -mu- kiambishi cha mtendewa, -khup- ni mzizi wa kitenzi, -ilkinyambulishi, -e ni kiambi tamati).
4. Mwa+ba+khup+il+e: mwabakhupile/mliwapiga (Mwa- nafsi ya pili wingi, -ba- kiambishi cha watendewa, -khup- ni mzizi wa kitenzi, -il- ni kinyambulishi, -e ni kiambishi tamati).

5. A+mu+khup+il+e: amukhupile/alimpiga (Anafsi ya tatu umoja, -mu- kiambishi cha kati kinachowakilisha aliyetendewa, -khup- ni mzizi wa kitenzi, -il- ni kinyambulishi, -e ni kiambishi tamati).

6. Ba+ba+khup+i+le: babakhupile/waliwapiga (Ba- nafsi ya tatu wingi, -ba- kiambishi cha kati kinachowakilisha waliotendewa, -khup- ni mzizi wa kitenzi).

\section{Khukholanila/Hali ya Kutendeana}

Katika hali ya kutendeana, kiishio cha kitenzi huongezewa -ne mwishoni mwake. Tazama mifano:

1. Na+khup+a+ne: Nakhupane/nilipigana Khwa+khup+a+ne: khwakhupane/tulipigana

2. Wa+khup+a+ne: wakhupane/ulipigana Mwa+khup+a+ne: mwakhupane/mlipigana

3. A+khup+a+ne: akhupane/alipigana Ba+khup+a+ne: bakhupane/walipigana 


\section{Khukholikha/Hali ya Kutendwa}

Katika hali ya kutendwa, kitenzi cha Kimasaaba huongezewa-ikhile. Tazama mifano:

1. Na+khup+ikhi+le: Nakhupikhile/nilipigwa Khwa+khup+ikhi+le: hwakhupikhile/tulipigwa

2. Wa+khup+ikhi+le:wakhupikhile/ulipigwa Mwa+khup+ikhi+le: mwakhupikhile/mlipigwa

3. A+khup+ikhi+le: akhupikhile/alipigwa Ba+khup+ikhi+le: bakhupikhile/walipigwa

\section{Khukholikhikha/Hali ya Kutendeka}

Katika hali ya kutendeka, kitenzi cha Kimasaaba huongezewa -ikhikha. Tazama mifano:

$$
\begin{aligned}
& \text { 1. Na+khup+ikhi+kha: Nakhupikhikha/nilipigika } \\
& \text { Khwa+khup+ikhikha: khwakhupikhi+kha/tulipigika } \\
& \text { 2. Wa+khup+ikhi+kha: wakhupikhikha/ulipigika } \\
& \text { Mwa+khup+ikhi+kha: mwakhupikhikha/mlipigika } \\
& \text { 3. A+khup+ikhi+kha: akhupikhikha/alipigika } \\
& \text { Ba+khup+ikhikha: bakhupikhikha/walipigika }
\end{aligned}
$$

\section{Kholisa/Hali ya Kutendesha}

Katika hali ya kutendesha, kitenzi cha Kimasaaba huongezewa -nisile. Tazama mifano:

1. Na+khupa+nisi+le: Nakhupanisile/nilipiganisha Khwa+khup+a+nisi+le: khwakhupanisile/tulipiganisha

2. Wa+khupa+nisi+le: wakhupanisile/ulipiganisha Mwa+khupa+nisi+le: mwakhupanisile/mlipiganisha

3. A+khupa+nisi+le: akhupanisile/alipiganisha Ba+khupa+nisi+le: bakhupanisile/ walipiganisha

\section{HITIMISHO}

Kwa kuzingatia matokeo ya utafiti huu, inabainika wazi kuwa lugha za Kiafrika hasa zile za Kibantu huwa zinaruhusu mnyambuliko wa vitenzi kutekelezwa. Kimasaaba kama lugha mojawapo ya Kiafrika huwa inakubali mnyambuliko wa vitenzi kufanyika. Mnyambuliko huu wa vitenzi huwa unawezesha mawasiliano kufanyika kwa urahisi. Hii ni kwa sababu wazungumzaji wa lugha wanaweza kutumia neno moja kuwasilisha maana tofauti tofauti. Wazungumzaji wa lugha wasipokua na uwezo wa kunyambua vitenzi, wao huwa wanakumbwa na matatizo katika mazungumzo yao. Mnyambuliko wa vitenzi ni stadi mojawapo ya kutumia lugha.

Utafiti huu unaonyesha kwamba lugha za Kiafrika zina rundo la mambo mazuri yanayohitaji kufanyiwa utafiti. Ni kweli kwamba lugha zetu si duni kama watu wengi wanavyofikiria. Ni jambo la kusikitisha kuona kwamba ni lugha chache sana za Kiafrika ambazo zimetafitiwa kufikia sasa. Kwa hiyo, wanaisimu na wanafasihi wanastahili kuangazia jambo hili. Ikiwezekana, CHAMA cha kuendeleza utafiti kuhusu lugha za Kiafrika kiundwe. CHAMA hiki kikiundwa, bila shaka kitaangazia lugha mbalimbali za Kiafrika na kuhakikisha kwamba kila lugha inashughulikiwa vilivyo. Ili jambo hili liweze kufanyika, kuna mahitaji ya nchi zetu za Kiafrika kuanzisha mfuko wa pesa za kufadhili CHAMA hicho. Hii inamaanisha kuwa muungano wa nchi za afrika (OAU) unastahili kuchukua hatua. Bila kufanya hivyo, lugha nyingi za Kiafrika zitaangamia na sote tutakuwa tukizungumza Kiingereza, Kifaransa, Kichina, Kiarabu na Kijerumani ambazo pia ni lugha za mama katika mataifa hayo. Hii ni kwa sababu Waafrika wengi wanachukulia lugha zao kuwa duni.

\section{MAREJELEO}

Baraka, Y. (2018). Unyambulishaji wa Vitenzi vya Lugha ya Kinyakyusakatika Mkoa wa MbeyaKyela. Morogoro, TZ: Chuo Kikuu cha Mtakatifu Augustino.

Katamba, F. (2003). Bantu nominal morphology. The Bantu languages, 103, 120.

Khamis, A. M. (2008). Maendeleo ya Uhusika, Taasisi ya Uchunguzi wa Kiswahili. Dar es Salaam: Taasisi ya Uchunguzi wa Kiswahili, Chuo Kikuu cha Dar es Salaam

Kihore, Y. M., Massamba, D. P. B., \& Msanjila, Y. P. (2003). Sarufi maumbo ya Kiswahili sanifu (SAMAKISA): Sekondari na vyuo. Taasisi ya Uchunguzi wa Kiswahili, Chuo kikuu cha Dar es Salaam.

Matei, A. K. (2008). Darubini ya sarufi : ufafanuzi kamili wa sarufi ya Kiswahili : maswali na mazoezi. Nairobi: Phoenix Publishers. 
Mbwillow, S. N. (2017). Uambikaji kama Dhana Kuu: Tofauti ya Uambikaji na Unyambulishaji. Chuo Kikuu cha Dar es Salaam.

Mhadhara wa 8 katika kitabu kilichotolewa na Chuo Kikuu Huria cha Tanzania mwaka wa (2008). Uambishaji na Mnyambuliko wa Maneno, Taasisi ya Elimu, Chuo Kikuu Huria cha Tanzania, Dar es Salaam.

Niyirora, E. \& Ndayambaje, L. (2012). Kiswahili Sanifu kwa Shule za Sekondari. Kitabu cha Mwalimu, Kidato cha Tano. Tanprints.

TUKI. (2004). Kamusi ya Kiswahili Sanifu. Nairobi, KE: OxfordUniversityPress.

Wahiga, G. (1999). Sarufi Fafanuzi ya Kiswahili. Nairobi, KE: Longhorn Publishers. 